\title{
Development of Semi-autonomous Control Modes in Laparoscopic Surgery Using Automatic Visual Servoing
}

\author{
Alexandre Krupa ${ }^{1}$, Michel de Mathelin ${ }^{1}$, Christophe Doignon ${ }^{1}$, \\ Jacques Gangloff ${ }^{1}$, Guillaume Morel $^{2}$, Luc Soler ${ }^{3}$, and Jacques Marescaux ${ }^{3}$ \\ 1 LSIIT (UPRES-A CNRS 7005), University of Strasbourg I, France \\ alexandre.krupa|michel. demathelin@ensps.u-strasbg.fr \\ 2 EDF R\&D, Chatou, Paris, France \\ 3 IRCAD (Institut de Recherche sur le Cancer de l'Appareil Digestif), Strasbourg.
}

\begin{abstract}
This paper presents ongoing research on the development of automatic control modes for robotized laparoscopic surgery. We show how visual feedback can be used in a control scheme to autonomously perform basic surgical subtasks in order to enhance surgeon accuracy and comfort.
\end{abstract}

\section{Objective}

Laparoscopic surgical robots have appeared recently. With these systems, robot arms are used to manipulate the instruments and the camera. The surgeon teleoperates the robots through master arms using the visual feedback from the laparoscope. This reduces the surgeon tiredness, and potentially increases accuracy by the use of a high master/slave motion ratio. Our research in this field is aimed at expanding the potentialities of such systems by providing "automatic modes" in which the system autonomously performs simple subtasks. For this purpose, the robot controller uses the visual feedback from the laparoscope to automatically drive instruments, through a visual servo loop, towards their desired location. In cooperation with IRCAD, we particularly focus on liver surgery. This surgery involves a number of repetitive gesture, such as the cleaning-suction process, clamping, cauterization, needle manipulation. It shall be noticed that although these processes are rather simple as compared to critical surgical gestures, it involves repetitive movements for the surgeon who drives master devices.

\section{Methodology and Ongoing Experiments}

A difficulty in laparoscopic surgery is the lack of depth information due to the monocular vision system. Furthermore, as surgical scenes are poorly structured,

\footnotetext{
${ }^{0}$ Aknowledgment: The experimental part of this work has been made possible thanks to the collaboration of Computer Motion that has graciously provided the medical robot.
} 
it is difficult to extract reliable reference points and visual features to be used in visual servoing techniques. In our approach, we add different structured lightening systems made of laser pointers mounted on a surgical tool-holder. The structured light allows for the robust extraction of visual features that can be used in our image-based control law. The endoscopic camera is mounted on another robot's arm whose relative position is unknown with respect to the other arms holding the surgical tool. This is a major difficulty for visual servoing, that we tackle by robustly identifying part of the Image Jacobian matrix expressing the relationship between visual displacements of the tool in the image around a working position and robot axis rotations. As an example, we can imagine that the surgeon select an area on the monitor screen to indicate a vessel to be reached. By indicating points on the screen, the surgeon specify the target point on the vessel and a desired orientation of the tool with respect to it. Then the projected position and orientation of the tool can be directly controlled with images of the impact laser features as follow (see, e.g., Fig. 1). - Firstly, a rotation around the tool axis is provided for alignment through visual servoing. - Then, laser features are moved onto the projection of the vessel by rotating the tool around the trocard center. - Finally, a simple motion along the tool axis aligns the tool marker with the laser points. We have already successfully tested this vision control with a 6 DOF robot, constrained to 4 DOF (using force feedback), on a laparoscopic trainer (see [1]). An automatic cleaning task has been implemented on a 4 DOF medical robot (Computer Motion) and tests on living animal have been performed.

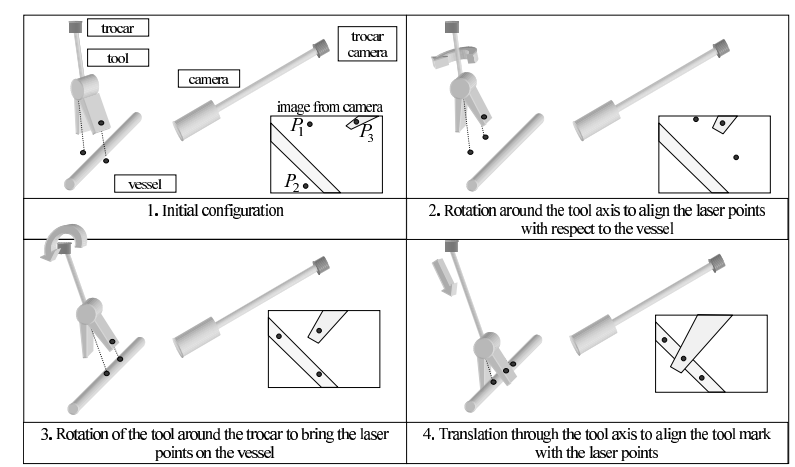

Fig. 1. Example of visual servoing using laser markers.

\section{References}

1. A. Krupa, C. Doignon, J. Gangloff, M. de Mathelin, L. Soler, G. Morel. Towards semi-autonomy in laparoscopic surgery through vision and force feedback control. Proc. of the Seventh International Symposium on Experimental Robotics (ISER), Hawaii, December, 2000. 